\title{
Design of New-type Impactor Based on Combined Effect by Air-pressure and Electromagnetism
}

\author{
Weizhang Wang ${ }^{1}$, Zengmei Song ${ }^{{ }^{*}}$, Lin Shi ${ }^{2}$ \\ ${ }^{I}$ Mechatronic \& Vehicle Engineering School of Weifang University, Weifang, Shandong China \\ ${ }^{2}$ Shandong Tianrui Heavy Industry cooperation Ltd., Weifang, Shandong China \\ *Corresponding Author.
}

\begin{abstract}
Analysis of rock-breaking mechanism of cyclical impact loading under high and low impact on the basis of impact stress wave theory holds that microcrack occurs in the rock due to high energy impact and the following medium and low impact can cause rapid destruction of the rock. Intermittent impact method of variable impact energy is hence suggested. Electromagnetically-propelled device is added to the traditional air hammer by means of intermittent intervention by electromagnetic impact. Combined impact by gas and power and interval pneumatic impact is applied to design new-type impactor based on the combined effect by air-pressure and electromagnetism, which is supposed to provide a new solution for improvement of impactor work efficiency.
\end{abstract}

Keywords: Stress wave, electromagnetism, air-pressure, impactor.

\section{Introduction}

The submersible drilling rig is mainly used in foundation anchor, mining and other operations where rock-drilling is needed. It has advantages of higher reliability, higher drilling efficiency, larger drilling diameter and larger drilling depth compared with ordinary rock drills. The impactor, responsible for drilling rock holes and discharging cuttings, is the actuator of the submersible drilling rig and determines its working performance[1].

Drilling in shallow strata nowadays are mainly performed by the aid of hydraulic impactors and air-pressure impactors. Because of low efficiency of hydraulic impactors, air-pressure impactors are generally adopted[2].

Most air-pressure impactors are driven by the high pressure air generated by the air compressor. The energy of the high-pressure air is used for driving the pit to transmit impact energy and break the rock, during which part of the air enters the bottom of the hole and blows the rock powder. Higher impact frequency and impact energy are generally raised to improve the drilling efficiency of the air-pressure impactor. The impact frequency and impact energy are related to the pressure of the high-pressure air provided, i.e, the higher air pressure and impact frequency, the larger impact energy. Higher impact frequency leads to more frequent movement of the pit and aggravates the pit abrasion, thus reducing the lifespan of the air-pressure impactor. Therefore traditional air-pressure impactors have to shorten their lifespan to gain higher drilling efficiency, or reduce the drilling efficiency to prolong the lifespan[3]-[4]. In addition, in spite of high efficiency, air-pressure drilling requires more of the compressor. Rated pressure of existing compressors can not meet the requirements of the impactors whereas speeding up drilling merely depends on increasing the air pressure.

This thesis presents a new type of impactor, combined impactor by air-pressure and electromagnetism, which can increase electromagnetic impact force on the basis of air impact and significantly raise the impact efficiency without additional air load.

ISSN: 0010-8189

(c) CONVERTER 2020

www.converter-magazine.info 


\section{Rock Breaking Mechanism}

A rock is a mineral aggregate of certain frame, stable shape and structure with rather strong mechanical stability. Rock breaking is related to its strength. The impactor can impact and break the rock because of application of cyclic dynamic load to impact the rock, causing stress damages and microcracks of the rock due to cyclic disturbance of the dynamic load. The microcracks expand because of the cyclic impact and finally cause breaking of the rock.

Impactor bit impacting rock mass can be calculated and analyzed by stress wave theory. The stress wave in the rock mass can be categorized as Rayleigh wave spreading along the outer free surface of the rock mass, and shear wave and expansion wave spreading inside the rock mass, as shown in Fig.1. The shear wave combined with expansion wave are called body wave, key to cracking of the rock body.

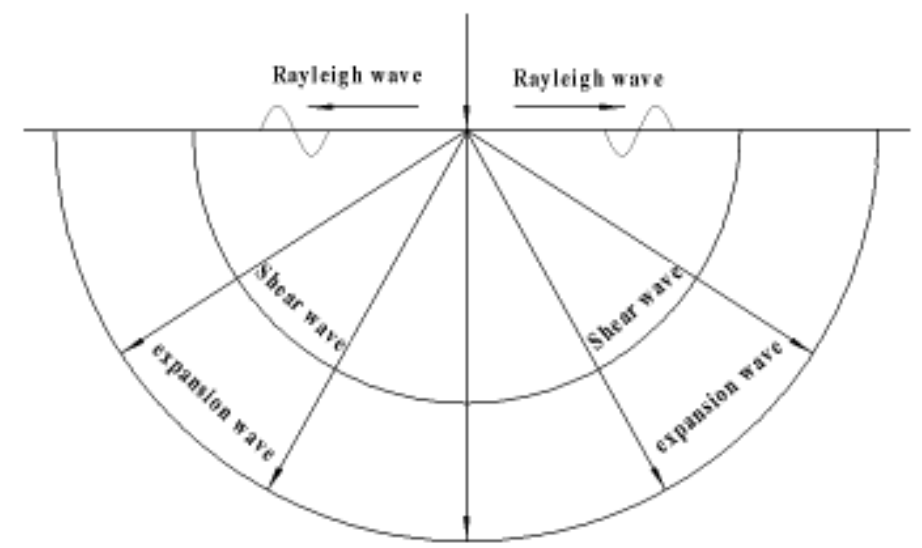

Fig 1: Distribution of stress wave in the rock mass when impacted

2.1 Relations between impact strength and damages of the rock

The cracking of the rock is related to the impact strength when the impactor works. Wang Zhiliang and Yang Hui[5] studied the dynamic mechanical properties of granite under cyclic impact loading. Using the improved experimental equipment, four different incident wave stress amplitudes(respective samples A1 110.57 MPa, B190.48 MPa, C1 $70.82 \mathrm{MPa}, \mathrm{D} 150.69 \mathrm{MPa}$ ) were applied to exert Constant amplitude cyclic impact on granite. Four rock samples with different incident wave stress amplitudes are extracted from the experimental results, and the stress-strain curves of rock mass under one (the first ) impact are shown in Fig.2.It can be seen from the figure that higher impact energy will cause higher peak stress and higher strain in the rock mass.
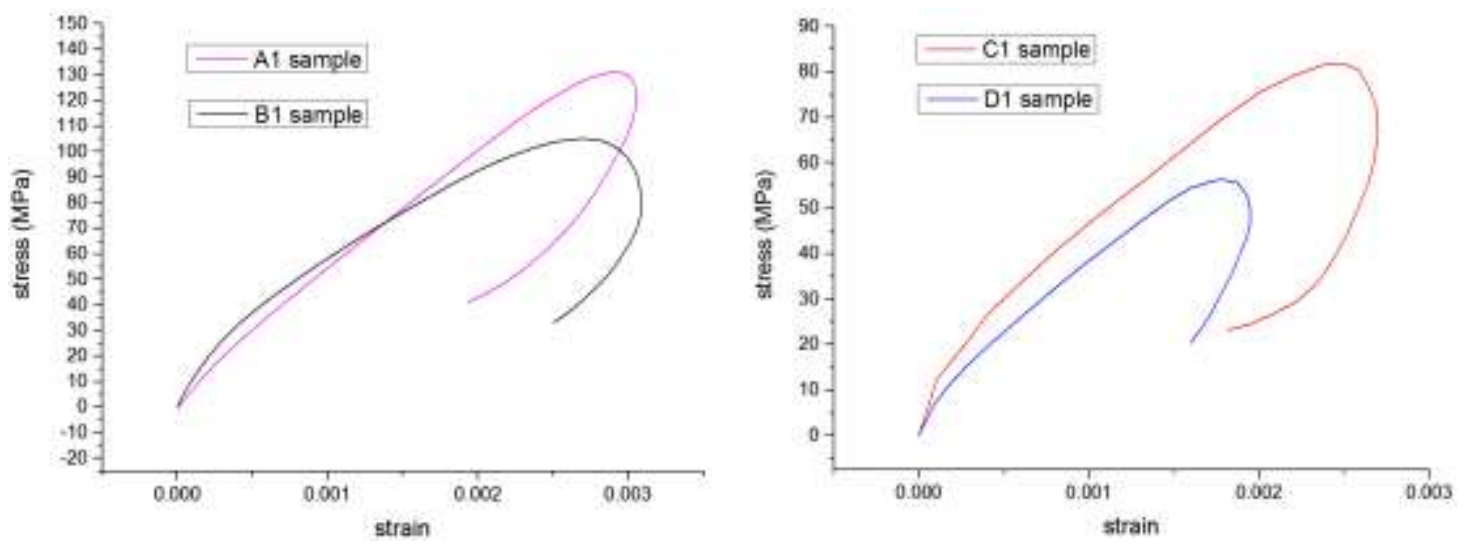

Fig 2: Stress-strain curve under single impact load

ISSN: 0010-8189

(C) CONVERTER 2020 
From the linear point of view, it is close to the linear elastic strain before reaching the peak stress, and there is a certain rebound after reaching the peak stress, without causing the macroscopic damage of rock mass.However, the peak stress plays a decisive role in the microscopic damage inside the rock mass, and the larger the peak stress is, the more favorable it is for rock breaking.Ge Xiurun[6] analyzed the fatigue damage law of rock under triaxial confining pressure by CT scanning from a microscopic point of view, and found that the magnitude of the peak stress provided by the applied cyclic impact force was closely related to the internal damage of rock mass. If the peak stress is lower than the threshold value of rock breaking in static test, the rock mass is basically not damaged by cyclic impact. If the peak stress is higher than the threshold value of static test, the rock mass is damaged and breaks rapidly with the increase of impact times. The higher the peak stress is, the higher the damage degree is and the less the impact times needed.

\subsection{Interval impact effect}

Here comes a question If the rock mass receives a high-strength impact, and the peak stress in the rock mass is higher than the rock breaking threshold value of the static test, then the medium-low strength impact is applied, the peak stress in the rock mass caused by this impact is slightly lower than the rock breaking threshold value of the static test. What will happen? Will the rock break?

The above discussion can be converted to the problem of rock mass bearing cyclic impact load with microcracks, as shown in Fig.3. When subjected to impact load, the stress wave spreads with the impact point as the center, and its radiation shape is semi-circular. When the stress wave radiates to the microcracks, the surrounding scattering will occur, and energy will accumulate at the tip opening of the microcracks where highly concentrated stress will be generated. When the stress at the tip opening exceeds the dynamic fracture toughness of the rock mass, considering the tensile effect of the unloading wave on the microcracks, the microcracks will be further torn and expanded.

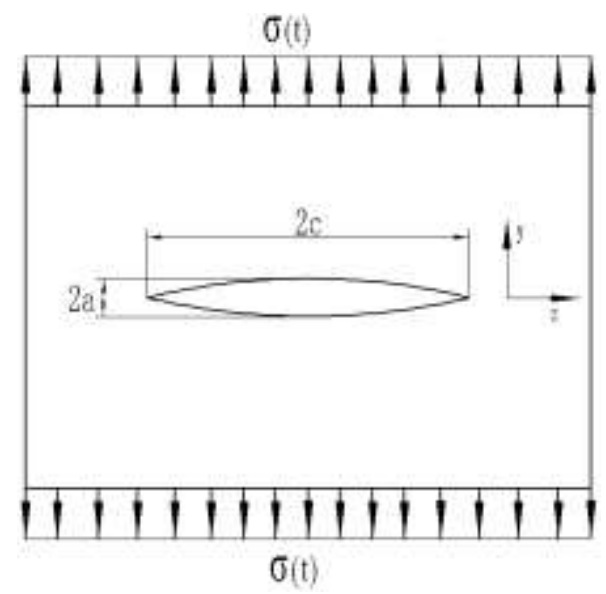

Fig 3: Shape of rock mass with micocracks (with confining pressure omitted)

It is necessary to calculate the dynamic stress intensity factor KIS(t) of microcracks to study whether microcracks extend and even whether rock mass breaks. When the stress intensity factor KIS(t) is greater than the dynamic fracture toughness (the critical value of stress intensity factor), the rock mass will break.

Green, Sneden and Irwin ${ }^{[7]}$ proposed that the open displacement of any point on the elliptical crack surface along the Y direction can be drawn from the stress and strain in the deep-buried elliptical crack neighborhood of elastic brittle materials in the calculation of brittle rock mass:

Where

$$
y_{0}=\frac{2 \cdot\left(1-\mu^{2}\right) \cdot \sigma \cdot a}{E \cdot \varnothing}
$$

ISSN: 0010-8189 


$$
\varnothing=\int_{0}^{\frac{\pi}{2}}\left[\sin ^{2} \varphi+\left(\frac{a}{c}\right)^{2} \cos ^{2} \varphi\right]^{\frac{1}{2}} \cdot d \varphi
$$

Whereby stress intensity factor is derived

$$
\mathrm{K}_{I}=\frac{\sigma \cdot \sqrt{\pi}}{\emptyset} \cdot\left(\frac{a}{c}\right)^{\frac{1}{2}} \cdot\left(c^{2} \cdot \sin ^{2} \varphi+a^{2} \cdot \cos ^{2} \varphi\right)^{\frac{1}{4}}
$$

The above is the solution of the stress intensity factorK ${ }_{I}$ of the static problem, but the dynamic problem of rock mass structure with cracks under impact load is more complex, and the interaction between the impact stress wave and the microcracks needs to be considered.

Based on the differences between the dynamic strength stress factor and the static strength factor, Nakano Motohiro and Kishida Keizo[8] proposed the dynamic correction coefficient $\partial(\mathrm{t})$. The impact correction function is calculated by the ratio of the unique dynamic value and the static value of the microcrack tip opening. The dynamic strength stress factor is finally obtained by the ratio of the static strength factor and the dynamic correction coefficient:

$$
\mathrm{K}_{\mathrm{IS}}(\mathrm{t})=\frac{\mathrm{K}_{\mathrm{I}}}{\partial(\mathrm{t})}
$$

At present, the calculation method of dynamic strength stress factor is widely used. The value of dynamic strength stress factor calculated by this method is much larger than that of static strength factor.

From the above analysis, it can be concluded that on the basis of high strength impact, the subsequent impact of medium and low strength will lead to rock breaking even if the peak stress of rock mass is slightly lower than the threshold value of rock failure in static test. However, due to the complexity of the problem, this thesis will no longer give quantitative analysis.

Based on the above principles, a pneumatic-electromagnetic combined impactor is proposed. Electromagnetic impact force is added on the basis of air pressure impact. Due to the intermittent characteristics of electromagnetic impact, this design implements intermittent intervention of electromagnetic impact. With electromagnetic impact intervention, its impact energy is greatly increased, and the amplitude of the incident wave stress during the impact is also greatly increased, making the internal stress peak value of the rock mass far exceed its breaking strength, resulting in lots of small cracks. In further low- and medium-strength impacts, the tip strength stress factor of the micro-cracks continues to increase under the action of the impact stress wave, and the micro-cracks further expand. Under the cyclic impact, numerous micro-cracks expand and then connect to each other, causing the rock mass to break.

\section{Impactor Structure Design}

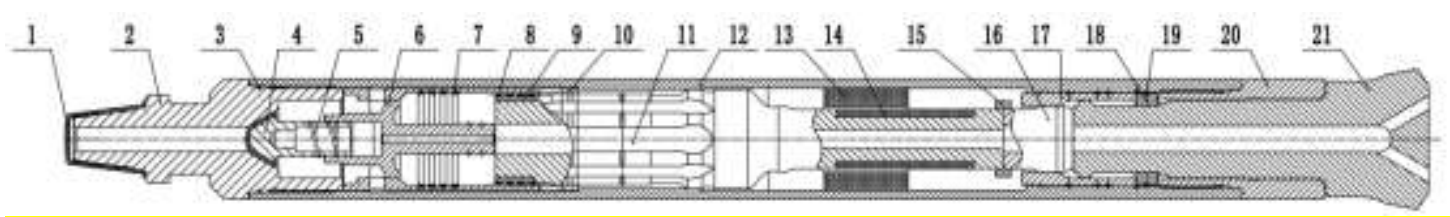

Fig 4: Schematic diagram of the structure of the pneumatic-electromagnetic combined impactor

In the figure: 1-rear dust cap, 2-rear joint, 3-first O-shape seal ring, 4-check valve, 5-spring, 6-air distributor, 7-buffer counter coil, 8-promoter magnetic pole, 9-promoter yoke, 10-inner cylinder, 11-piston, 12-outer cylinder, 13-drive coil, 14-driven coil, 15-piston connecting sleeve, 16-changeable piston head, 17-guide sleeve, 18-snap ring 19-second $O$-shape seal ring, 20-front joint, 21-drill.

The technical scheme adopted in this design is shown in Fig.4. The outer cylinder is equipped with an air distributor, an inner cylinder, a guide sleeve, a drive coil and a piston. The piston can slide inside the outer cylinder; both ends of the outer cylinder are respectively connected with the front joint and rear joint using thread; the inner cylinder is

ISSN: 0010-8189

(C) CONVERTER 2020 
equipped with a buffer counter coil; the piston is installed with the promoter magnetic pole, promoter yoke, driven coil, piston connecting sleeve, and replaceable piston head. The rear joint is covered with a rear dust cap. The rear joint connection is sealed with a first O-shape seal ring, and the rear joint is connected to a check valve. The check valve is connected to the air distributor through a spring. The inner wall of the inner cylinder is equipped with buffer counter coil, and the piston is equipped with a promoter pole at the back. The promoter magnetic pole is provided with a promoter yoke; the driven coil is installed in the middle of the piston, and the drive coil is installed on the inner wall of the outer cylinder. The piston is connected to the replaceable piston head through the piston connecting sleeve, and the replaceable piston head impacts the drill under guidance of guide sleeve. The drill is covered with a snap ring and a second $\mathrm{O}$-shape seal ring.

\section{Working Principle}

The pneumatic-electromagnetic combined impactor is added with an electromagnetic propulsion device on the basis of the traditional pneumatic impactor, so that the piston is subjected to an electromagnetic driving force much greater than the pneumatic driving force, thus transmitting greater impact energy. The drive coil discharges at an interval of certain impact times, which improves drilling efficiency and saves energy. The new piston connects the piston and the replaceable piston head through the piston connecting sleeve, which can be easily replaced when the replaceable piston head is damaged.

The impact method adopts pneumatic-electromagnetic combined impact and pneumatic impact with intervals, that is, $\mathrm{N}$ times of pneumatic impact after one pneumatic-electric combined impact. In the stroke stage, the buffer counter coil is energized when the promoter magnetic pole and the promoter yoke are about to leave the buffer counter coil, thus giving electromagnetic force to the piston. When the driven coil is about to leave the drive coil, the drive coil is energized to give the piston electromagnetic force. When the promoter magnetic pole and the promoter yoke are about to approach the buffer counter coil in return stroke, the buffer counter coil generates induced current to energize the large capacitor of connecting drive coil. Meanwhile, the piston decelerates.

The starting position is at the moment when the piston hits the drill, and the right side is the forward direction. Pushed by the high-pressure gas, the piston moves backward and enters the return phase. At this stage, the drive coil and the driven coil do not work; when the permanent magnet at the piston tail passes through the buffer counter coil, an induced current is generated in the buffer counter coil to charge the capacitor of the drive coil. Meanwhile, the promoter magnetic pole is subjected to the reaction force of the induced magnetic field generated by the buffer counter coil, which then decelerates and stops.

After the control system receives the induced current, it records the number of round trips of the piston. When the number of round trips reaches $\mathrm{N}$ times, the capacitor of the drive coil is controlled to discharge electricity; if it is less than $\mathrm{N}$ times, the drive coil still does not work; if the piston has $\mathrm{N}$ times of round trips, the control system starts timing; the piston is pushed forward by the high-pressure gas, the control system controls energization of the buffer counter coil, the promoter magnetic pole is accelerated under the thrust; when the piston moves to the set position, the control system stops timing, and the large capacitor connected to the drive coil discharges, generating pulse current. Such current has a high peak value and short duration, which generates strong induced magnetic field. The piston receives a great thrust and gains great kinetic energy; the replaceable piston hits the drill to release the impact energy, and the drill impacts the rock to produce a large amount of micro cracks. The subsequent small-energy impact causes crack spreading and rock breakage. The high-speed gas blown out of the drill exhaust hole continuously blows away the rock dust, so that a working cycle is completed.

\section{Conclusion}

Under high-intensity impact, even if the subsequent impact of medium and low intensity makes the peak stress in the rock mass slightly lower than the rock failure threshold value of the static test, the rock mass will be broken.

ISSN: 0010-8189

(C) CONVERTER 2020 
2. The electromagnetic impact force is added on the basis of air pressure impact. The impact energy is then greatly increased, and so is the incident wave stress amplitude during impact, which makes the internal stress peak of the rock mass far exceed its breaking strength, resulting in a large number of small cracks.

Adopting the pneumatic-electromagnetic combined impact and the pneumatic interval impact, the designed new-type impactor based on pneumatic-electromagnetic combined action can significantly improve the impactor working efficiency.

\section{References}

[1] X.M. Chen, W. Liu, "Application of ZGYX460 Open-pit Drilling Rig in Chengmenshan Copper Mine," Copper Engineering, vol. 35, no. 1, pp. 119-121, 2019.

[2] S. Fei, "Progress and trend of underground metal mining technology and down the hole drilling machine," China Metal Bulletin, vol. 26, no. 3, pp. 30-31, 2019.

[3] N. Qi, L. Chen, "Application of wheel walking and steering system in down-the-hole drill," Construction Machinery \& Maintenance, vol. 26, no. 3, pp.47-48, 2020.

[4] S.H. Xu, Huiian, "Shu Discussion on automatic anti sticking control in rock drilling process of down the hole drill," Internal Combustion Engine \& Parts, 2019, no. 12, pp.272-273, 2019.

[5] Z.L. Wang, H. Yang, N.C. Tian, "Mechanical properties and damage evolution mechanism of granite under uniaxial cyclic impact," Journal of Harbin Institute of Technology, vol. 52, no. 2, pp.59-65, 2020.

[6] X.R. Ge, J.X. Ren, Y.B. Pu. Preliminary study on CT meso-analysis of the law of stone fatigue damage expansion. Chinese Journal of Geotechnical Engineering. vol. 23, no. 2, pp. 191, 2001.

[7] C.K. Shen, Fracture mechanics, Tongji University Press. Shanghai 1996.03

[8] N. Motohiro, K. Keizo, "Numerical Calculation of Dynamic Stress Expansion Coefficient Based on Impact Failure Experiment,” Journal of the Japanese Society of Aeronautics and Astronautics., vol. 36, no. 410, pp.147-154, 1988. 